\title{
A Macaulay2 interface for Normaliz
}

\author{
WINFRIED BRUNS AND GESA KÄMPF
}

\begin{abstract}
Normaliz is a tool for the computation of Hilbert bases of normal affine monoids and related tasks. We describe the Macaulay2 interface to Normaliz. It makes Normaliz accessible for operations on monoid algebras in Macaulay2 and, conversely, makes Macaulay 2 a front-end for Normaliz.
\end{abstract}

The PACKAGE And its ApPliCATIONS. Normaliz [BIS] solves a task that can be described from a geometric and an (equivalent) algebraic perspective. The objects of discrete geometry on which Normaliz works are finitely generated rational cones $C$, i.e., subsets of a space $\mathbb{R}^{d}$ consisting of the linear combinations $a_{1} x_{1}+\cdots+a_{n} x_{n}$ of an integral system of generators $x_{1}, \ldots, x_{n} \in \mathbb{Z}^{d}$ with nonnegative coefficients $a_{i}$. If $C$ is pointed $(x,-x \in C \Longrightarrow x=0)$, then the monoid $M=C \cap \mathbb{Z}^{d}$ has a unique finite minimal system of generators $\operatorname{Hilb}(M)$, called its Hilbert basis; see [BG] for the terminology and mathematical background. The computation of Hilbert bases is the primary goal of Normaliz. For information on its algorithms see [BI].

By the theorem of Minkowski and Weyl, $C$ is a (rational) finitely generated cone if and only if it is the set of solutions of a linear homogeneous system of inequalities (with rational coefficients). Therefore, the computation of Hilbert bases is equivalent to solving such a system of inequalities. For its input, Normaliz accepts systems of generators as well as systems of inequalities.

Normaliz got its name from the first task for which it was designed, namely the computation of normalizations of affine monoids, i.e., finitely generated submonoids $N=\mathbb{Z}_{+} x_{1}+\cdots+\mathbb{Z}_{+} x_{n}$ of a lattice $\mathbb{Z}^{d}$. An element $y \in \mathbb{Z}^{d}$ is integral over $N$ if $k x \in N$ for some $k \in \mathbb{Z}, k>0$. The integral closure $\widehat{N}$ of $N$ in $\mathbb{Z}^{n}$ is the monoid of all elements $x \in \mathbb{Z}^{d}$ that are integral over $N$. Geometrically it is given by $\widehat{N}=C \cap \mathbb{Z}^{d}$ for the cone $C=\mathbb{R}_{+} x_{1}+\cdots+\mathbb{R}_{+} x_{n}$. The normalization $\bar{N}$ of $N$ is $\bar{N}=C \cap \mathbb{Z} N$, and since $\mathbb{Z} N$ is isomorphic to a lattice $\mathbb{Z}^{r}$, the computation of $\bar{N}$ amounts to a Hilbert basis computation. Depending on an input parameter, Normaliz computes $\operatorname{Hilb}(\widehat{N})$ or $\operatorname{Hilb}(\bar{N})$.

Our terminology has been chosen to be compatible with that of commutative algebra: after the choice of a field $K$, we can consider the monoid algebra $S=K[N]$, its integral closure $\widehat{S}$ in the Laurent polynomial ring $K\left[\mathbb{Z}^{d}\right]$, and its normalization $\bar{S}$. Since $\widehat{S}=K[\widehat{N}]$ and $\bar{S}=K[\bar{N}]$, the computation of integral closures of monoid algebras is reduced to the consideration of the underlying monoids.

The package Normaliz provides direct access to Normaliz as well as ring theoretic functions applied to monomial subalgebras and monomial ideals in polynomial rings.

Direct aCCESS to Normaliz. The input to Normaliz is composed of matrices of integers. The interpretation of each matrix depends on a parameter called type.

2010 Mathematics Subject Classification. 13P99, 14M25, 52B20.

Normaliz version 2.0. 
In types 0 and 1 , the rows of the input matrix are interpreted as the generators of a monoid $N$. In type 0 , Normaliz computes $\operatorname{Hilb}(\widehat{N})$, and in type 1 it computes $\operatorname{Hilb}(\bar{N})$ (notation as above).

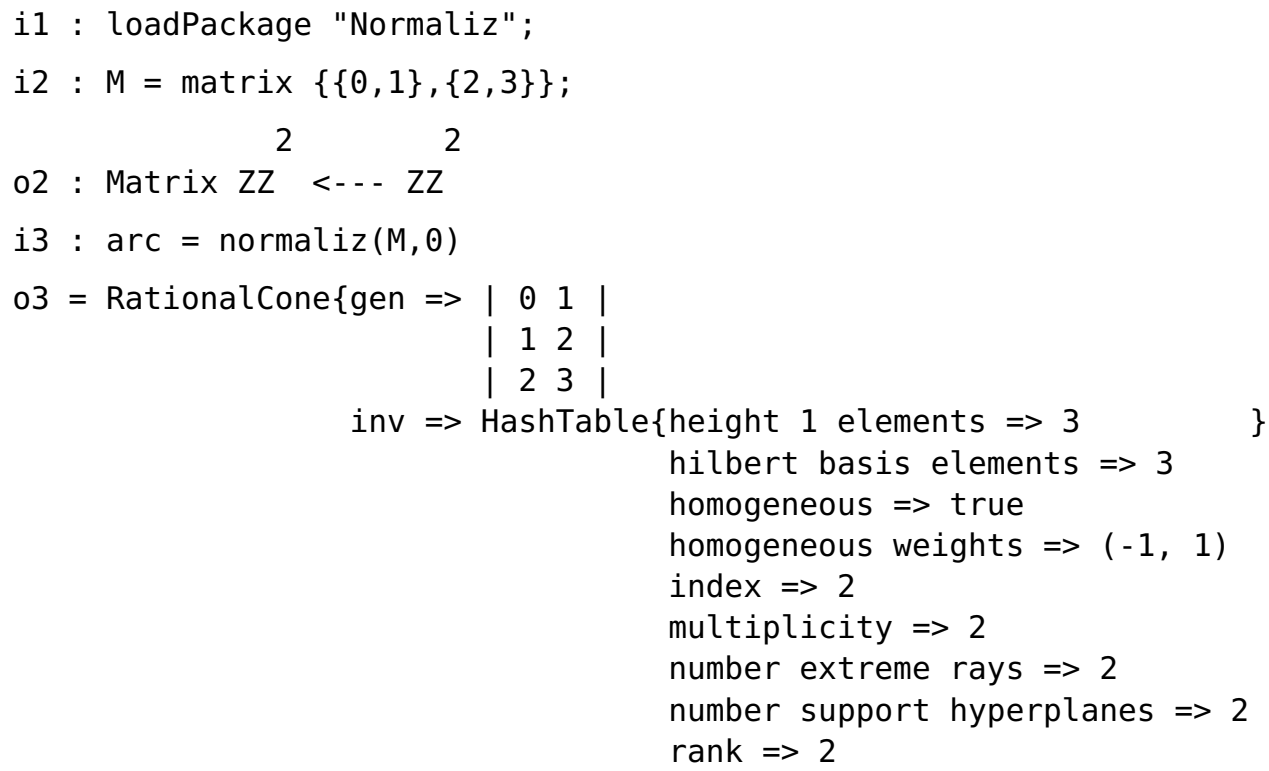

03 : RationalCone

The return value of normaliz is an object of type rationalcone defined by the package. Such an object is a hash table whose components are matrices and a hash table. The matrices are named after the suffixes of the output files of Normaliz from which the corresponding matrix is read. This example represents the minimal content of the rationalCone returned, namely the Hilbert basis in gen and the numerical invariants in inv.

The amount of data returned can be increased by the option allComputations:

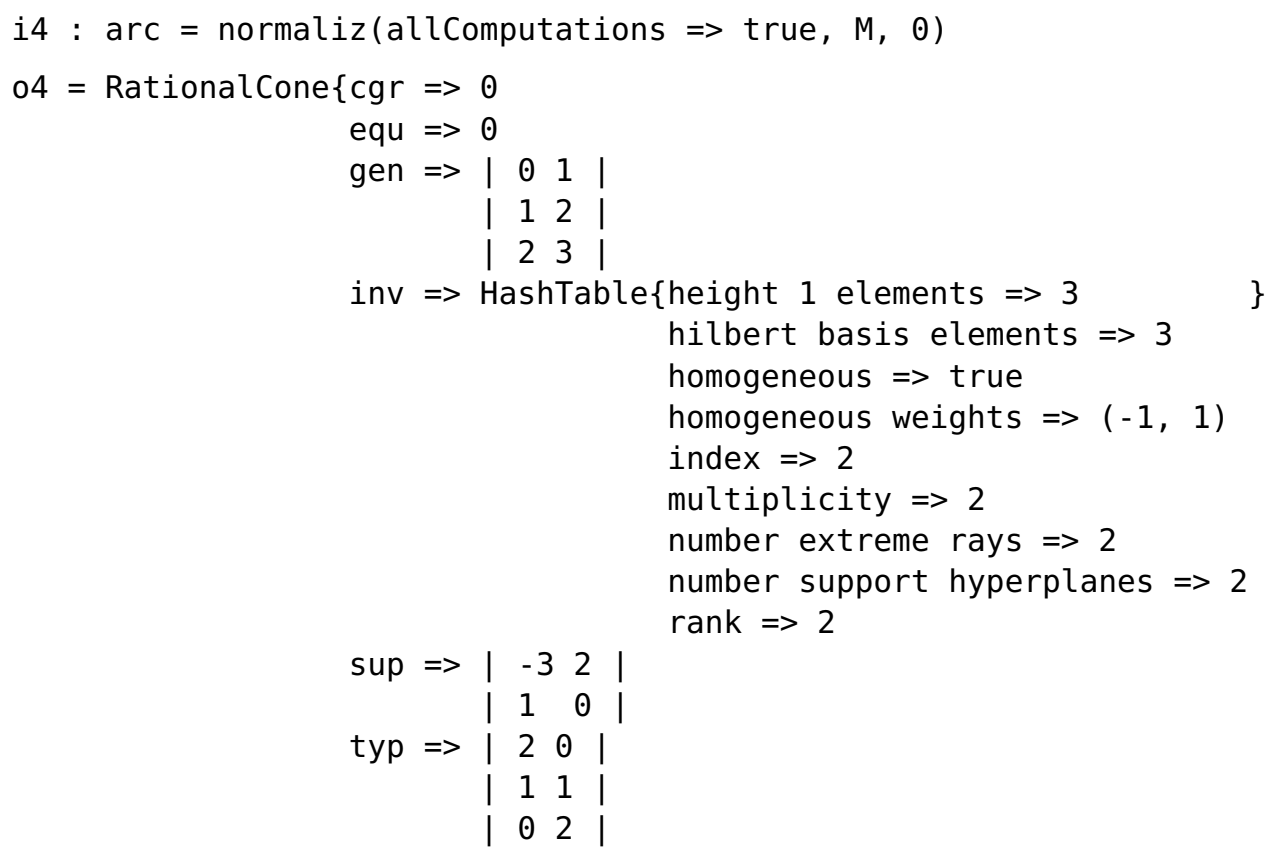


In addition to the data above, the rational cone will now contain four more components: cgr, equ, sup, and typ. The matrix arc\#" sup" contains the linear forms defining the cone. Each row $\left(\alpha_{1}, \ldots, \alpha_{d}\right)$ represents an inequality $\alpha_{1} \xi_{1}+\cdots+\alpha_{d} \xi_{d} \geq 0$ for the vectors $\left(\xi_{1}, \ldots, \xi_{d}\right)$ of the cone. The matrix arc\#"typ" contains the values of these linear forms on the Hilbert basis elements. The matrices arc\#"cgr" and arc\#"equ" are empty in this example: they contain the equations and congruences that together with the inequalities define the cone and the lattice, respectively, and in this example the inequalities are sufficient.

The output data of Normaliz are further increased by setNmzOption ( "allf", true). For the complete list of data and their interpretation see the Normaliz documentation or the package online help.

Normaliz has 6 more input types. Types 4, 5 and 6 allow the user to specify the cone and the lattice by homogeneous diophantine equations, inequalities and congruenences. A type 4 matrix is interpreted as a system of inequalities, defining the cone $C$ as just explained in connection with the matrix "sup". The rows $\left(\alpha_{1}, \ldots, \alpha_{d}\right)$ of a type 5 matrix are considered as equations $\alpha_{1} \xi_{1}+\cdots+$ $\alpha_{d} \xi_{d}=0$. In type 6 the matrix is interpreted as a system of homogeneous congruences, and $\mathbb{Z}^{d}$ is then replaced by the lattice of solutions. Types 4, 5 and 6 can be combined, and therefore the function normaliz has a variant in which the argument of the function is a list consisting of pairs (matrix, type). (The default type 4 matrix is the unit matrix, defining the positive orthant.)

For these types, setNMz0ption ("dual", true) chooses an alternative algorithm. It is often better than the triangulation based standard algorithm of Normaliz.

The input types 2 and 3 are variants of type 0 . In type 2 the rows $x_{1}, \ldots, x_{n}$ of the matrix are interpreted as the vertices of a lattice polytope, and type 0 is applied to the cone generated by $x_{i}^{\prime}=\left(x_{i}, 1\right) \in \mathbb{R}^{d+1}, i=1, \ldots, n$. Types 3 and 10 have a ring theoretic flavor. They will be explained in the next section. (Types 7, 8 and 9 are reserved for future extensions.)

Via suitable options one can restrict the data that Normaliz computes (see online help or Normaliz documentation). The extension setNmzOption ( "hilb" , true) is more important. It asks Normaliz to find the Hilbert series and polynomial of the (algebra over the) integral closure or normalization computed, provided a homogeneity condition is satisfied: there is an integral linear form $\lambda$ on $\mathbb{Z}^{d}$ or $\mathbb{Z} N$, respectively, such that $\lambda(x)=1$ for the extreme integral generators $x$ of the cone. For the introductory example, the data in arc\#" inv" now contain

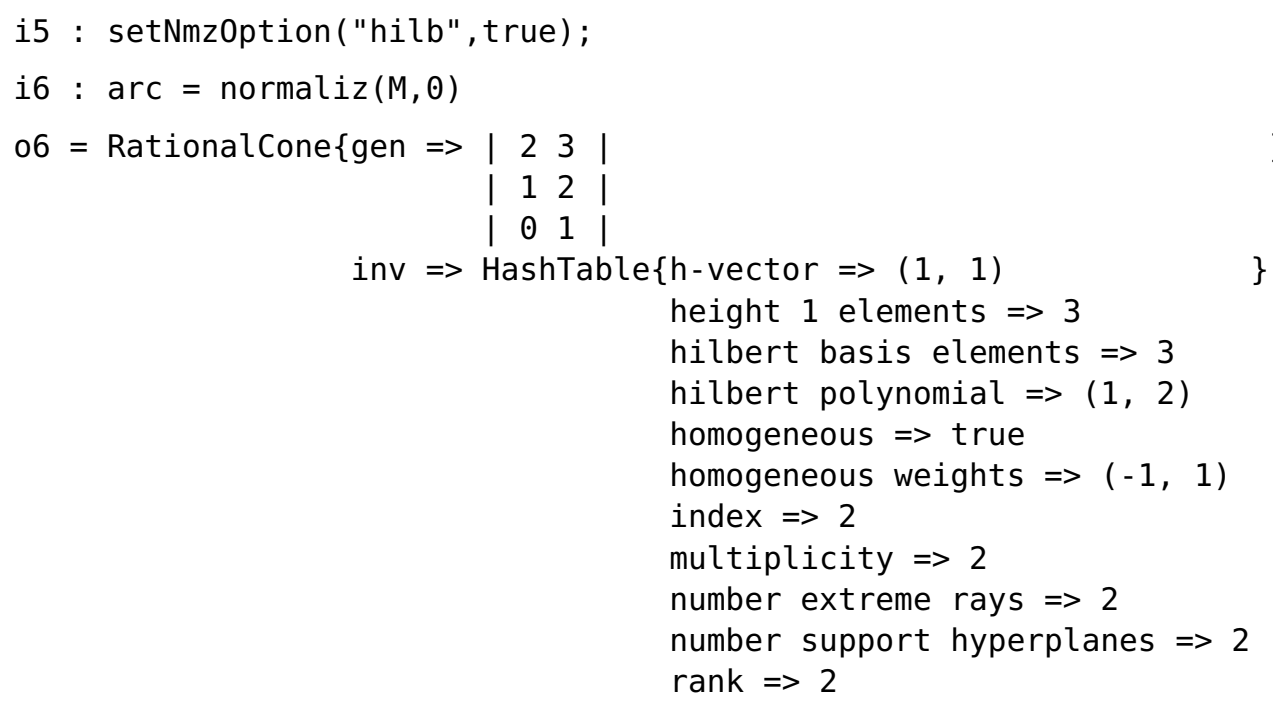


06 : RationalCone

The $h$-vector represents the numerator polynomial $1+t$ of the Hilbert series, and the Hilbert polynomial is $1+2 k$ where $k$ denotes the degree.

RING THEORETIC FUNCTIONS. For the ring theoretic functions the package introduces the class monomialsubalgebra. It is a subclass of Ring. A monomial subalgebra is created as follows:

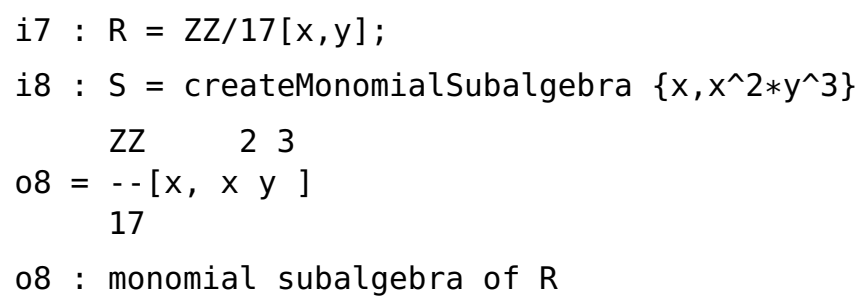

This creates the monomial $K$-subalgebra $S$ generated by the monomials $x, x^{2} y^{3}$ in the polynomial ring $R=K[x, y]$ over the base field $K$.

The functions normalToricRing and intclToricRing have monomial subalgebras as input (or just lists of monomials):

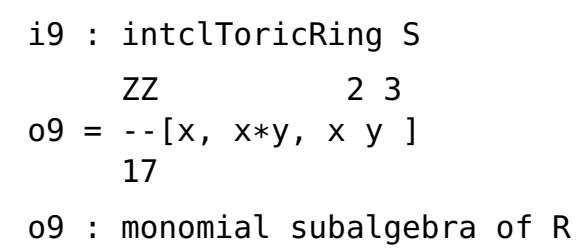

normalToricRing returns the normalization of $S$ as a monomial subalgebra of $R(R$ always contains the normalization), whereas intclToricRing returns the integral closure of $S$ in $R$ (or, equivalently, in the field of fractions of $R$ ). The cache of the returned monomial subalgebras contains the rational cone computed by Normaliz.

The function intclMonIdeal has a monomial ideal $I$ as its input. It computes the integral closure $\bar{I}$ of $I$ and the normalization of the Rees algebra of $I$. Consequently the return value is a sequence containing $\bar{I}$ (of type ideal) and a monomial subalgebra. Note that the Rees algebra $R[I t]$ and its normalization live in the extended polynomial ring $R[t]$. The function creates this extended polynomial ring, choosing an available name for the auxiliary indeterminate $t$. If $R$ itself is of type $R^{\prime}[t], I$ is a monomial ideal in $R^{\prime}$ and the normalization of the Rees algebra $R^{\prime}[I t]$ is to be computed, then the user can indicate this fact by adding the name of $t$ to the input.

The function intclToricRing calls Normaliz in type 3, made exactly for the computation of normalizations of Rees algebras.

The function normalToricRing has a variant in which the input parameter is an ideal $I$ consisting of binomaials $X^{a}-X^{b}$. It has a unique minimal prime ideal $P$ generated by binomials of the same type, and the function returns the normalization of $R / P$ embedded into a newly created polynomial ring of the same Krull dimension. (In general there is no canonical choice for such an embedding.)

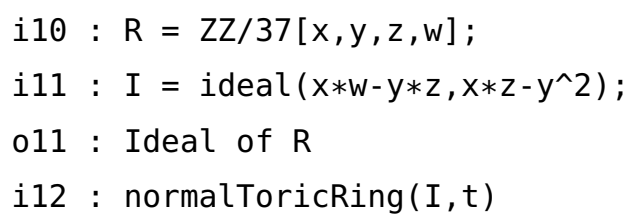




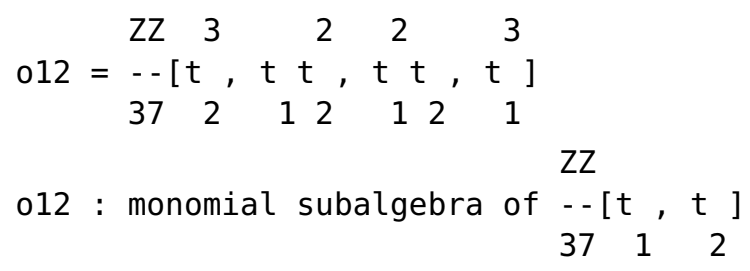

This function uses type 10 of normaliz, created exactly for this purpose.

There are further ring theoretic functions in the library including intersectionValRings, intersectionValRingIdeals, torusInvariants, finiteDiagInvariants and diagInvariants. The first two compute intersections of monomial valuation rings and ideals with the polynomial ring $R$ whereas the last three compute the rings of invariants of a diagonal torus action on $R$, a diagonal finite group action, or a diagonal group action in general.

MiscellaneA. Macaulay2 and Normaliz exchange data via hard disk files. By default the package handles the files behind the scenes, and the user need not care about them. However, the user can take over command of the file handling by specifying a file name and a path to the directory where the files are to be stored. The package provides functions for writing and reading Normaliz files directly. See the online help for details.

The standard integer precision of Normaliz is 64 bit (corresponding to the $\mathrm{C}$ integer type long long). Already in small dimensions this may not be sufficient. In that case one can choose the indefinite precision executable by setting nmzVersion="normbig". This choice typically increases the computation time by a factor of 5 . It is less time consuming to use setNmzOption ( "errorcheck" , true) in order to control the arithmetic of the 64 bit computation. For further functions and options we refer the reader to the online help.

ACKNOWLEDGEMEnTs. The authors are grateful to Dan Grayson for his support in the development of the Normaliz package.

\section{REFERENCES.}

[BG] W. Bruns and J. Gubeladze, Polytopes, rings, and $K$-theory, Springer Monographs in Mathematics, Springer, Dordrecht, 2009.

[BI] W. Bruns and B. Ichim, Normaliz: Algorithms for affine monoids and rational cones, J. Algebra 324 (2010), no. 5, $1098-1113$.

[BIS] W. Bruns, B. Ichim, and C. Söger, Normaliz, computing normalizations of affine semigroups, available at www. math.uos.de/normaliz.

[M2] D.R. Grayson and M.E. Stillman, Macaulay 2, a software system for research in algebraic geometry, available at www. math. uiuc. edu/Macaulay2/.

RECEIVED : 2009-03-23 REVISED : 2010-07-12 ACCEPTED : 2010-08-08

wbruns@uos. de : FB Mathematik/Informatik, Universität Osnabrück, 49069 Osnabrück, Germany.

gkaempf@uos.de : FB Mathematik/Informatik, Universität Osnabrück, 49069 Osnabrück, Germany. 